\title{
Clinical Management of Drug Resistant Epilepsy: A Review on Current Strategies
}

\author{
Deborah Guery' \\ Sylvain Rheims iD ${ }^{1-3}$ \\ 'Department of Functional Neurology \\ and Epileptology, Hospices Civils De \\ Lyon and University of Lyon, Lyon, \\ France; ${ }^{2}$ Lyon's Neuroscience Research \\ Center, INSERM UI028/CNRS UMR \\ 5292, Lyon, France; ${ }^{3}$ Epilepsy Institute, \\ Lyon, France
}

\begin{abstract}
Drug resistant epilepsy (DRE) is defined as the persistence of seizures despite at least two syndrome-adapted antiseizure drugs (ASD) used at efficacious daily dose. Despite the increasing number of available ASD, about a third of patients with epilepsy still suffer from drug resistance. Several factors are associated with the risk of evolution to DRE in patients with newly diagnosed epilepsy, including epilepsy onset in the infancy, intellectual disability, symptomatic epilepsy and abnormal neurological exam. Pharmacological management often consists in ASD polytherapy. However, because quality of life is driven by several factors in patients with DRE, including the tolerability of the treatment, ASD management should try to optimize efficacy while anticipating the risks of drug-related adverse events. All patients with DRE should be evaluated at least once in a tertiary epilepsy center, especially to discuss eligibility for non-pharmacological therapies. This is of paramount importance in patients with drug resistant focal epilepsy in whom epilepsy surgery can result in long-term seizure freedom. Vagus nerve stimulation, deep brain stimulation or cortical stimulation can also improve seizure control. Lastly, considering the effect of DRE on psychologic status and social integration, comprehensive care adaptations are always needed in order to improve patients' quality of life.
\end{abstract}

Keywords: drug resistant epilepsy, epilepsy surgery, antiseizure drugs, comprehensive care

\section{Definition of Drug Resistant Epilepsy}

Drug resistant epilepsy (DRE) is defined by the International League Against Epilepsy (ILAE) as the persistence of seizures despite at least two syndromeadapted antiseizure drugs (ASD) used at efficacious daily dose. ${ }^{1}$ According to this definition, the single variable that should be considered is whether or not the patient is seizure-free. In contrast, neither the type of seizures, the seizure frequency nor the other epilepsy-related complications are included in this definition. However, the latter drive the handicap resulting from DRE and its consequence on quality of life (QOL) at the individual patient level and will be considered in the therapeutic management of DRE.

Several issues need to be underscored:

- The minimum duration required to evaluate the response to an ASD might vary across patients, especially depending on the baseline seizure frequency. For a patient with several seizures per week, a follow-up of several weeks will be enough to conclude on ASD failure if seizures persist. In contrast, the evaluation period will be much longer for a patient with 1-2 seizures per year.
Correspondence: Sylvain Rheims Department of Functional Neurology and Epileptology, Hospices Civils De Lyon and University of Lyon, 59 Boulevard Pinel,

Bron Cedex, 69677, France

Tel +33472357900

Email sylvain.rheims@chu-lyon.fr 
- The threshold of two ASD may look arbitrary: it is related to the patterns of treatment response reported in prospective cohorts of epilepsy. ${ }^{2-4}$ The probability of seizure-freedom after two ASD failures decreases exponentially and is less than $5 \%$ after four failures. ${ }^{4,5}$ It should however be reminded that spontaneous or treatment-related seizure-free period of a minimum 12 months duration is reported in almost $20-25 \%$ of adult with DRE, with an annual rate of approximately $5 \%{ }^{6,7}$ When the analysis is limited to patients not eligible for epilepsy surgery, the cumulative probability of achieving a 12-month seizure remission period is of $33 \%$ at 7 years. ${ }^{8}$ Although this remission is typically transient, with $71 \%$ of patients relapsing within 5 years, ${ }^{8}$ these data might comfort the possible benefit at individual patient level of continuing to modify the ASD regimen, even after several failures. This might particularly be relevant for patients with frequent generalizedtonic seizures, especially considering the potential impact of this active management on the risk of sudden unexpected death in epilepsy (SUDEP). ${ }^{9}$

- Only half of patients with uncontrolled seizures might meet the ILAE criteria of DRE, ${ }^{10}$ suggesting the importance of re-evaluating patient's epilepsy history before conclusion. In a study evaluating the interrater agreement of the ILAE DRE definition between members of an epilepsy expert panel and individual investigators, $19 \%$ of patients were classified as having DRE by the investigators while considered to have "undefined responsiveness" by the expert panel. ${ }^{11}$ These divergences were mostly related to ASD dosage and/or the choice of the previously failed ASD.

- ASD prematurely withdrawn before having been titrated up to efficacious dose because of adverse events are not be considered as a failure for DRE criteria.

- Distinguishing drug-resistance from "pseudoresistance":

- Treatment compliance needs to be systematically evaluated. ${ }^{12}$ Beliefs about medications, mood or anxiety disorders, recent uncontrolled seizures, multiple dosage schedule, poor physician-patient relationship are factors of poor adherence. ${ }^{12}$

- Psychogenic non-epileptic seizures possibly lead to 20 to $25 \%$ of pseudo-resistance. ${ }^{13}$ This issue reinforces the importance of detailed diagnostic work-up in patients who develop drug-resistance, including long-term video-EEG when the description of the ictal semiology is not clear enough to exclude the occurrence of psychogenic nonepileptic seizures. This is particularly relevant in patients who suffer from both epileptic and psychogenic non-epileptic seizures.

- Using inefficacious, or even worsening, ASD for a dedicated epilepsy syndrome can also result in "pseudo-resistance". This issue has mostly been observed in patients with idiopathic generalized epilepsy, including childhood/adolescent absence epilepsy or juvenile myoclonic epilepsy, in whom some ASD, such as carbamazepine, phenytoin or gabapentin, can aggravate seizure frequency or precipitate status epilepticus. ${ }^{14}$ Importantly, distinguishing idiopathic generalized epilepsy from focal epilepsy may not be trivial in daily clinical practice. Focal symptoms can be reported at the onset primary generalized epilepsy seizures. ${ }^{15}$ Furthermore, focal EEG abnormalities can be observed in primary generalized epilepsies ${ }^{16}$ whereas generalized EEG features have been reported in $20-67 \%$ of frontal lobe seizures. ${ }^{17}$

\section{Epidemiology and Risk Factors}

The prevalence of DRE is $30 \%$ (95\% CI: $19-42 \%)$. Its incidence varies from 15\% (95\% CI: $11-19 \%)$ in children to $34 \%$ (95\% CI: $6-62 \%$ ) in adults ${ }^{18}$ without variation across geographic areas. Among patients with newly diagnosed epilepsy followed for at least two years in the Glasgow cohort, $36 \%$ were not seizure free the last year of follow-up. ${ }^{5}$ The response to treatment was dynamic, with $37 \%$ of patients achieving immediate seizure freedom at the beginning, $22 \%$ suffering from initial relapses before persistent seizure-freedom, $16 \%$ with long-term DRE despite periods of transient remission. ${ }^{2}$ Overall, $25 \%$ of patients were always drug resistant. ${ }^{2}$ Despite the increasing number of available ASD, this picture has remained unchanged. The long-term follow-up of the Glasgow cohort thus showed that the proportion of newly diagnosed patients who developed DRE has not been modified over the past twenty years, at $64 \%$ in 2000 and $63.7 \%$ in 2018 , despite the continual increase in the use of the new ASDs. ${ }^{5}$ 
Table I Risk Factors for Developing Drug Resistant Epilepsy

\begin{tabular}{|l|c|}
\hline Risk Factors & $\begin{array}{c}\text { Odd Ratio (95\% Confidence } \\
\text { Interval) }\end{array}$ \\
\hline Age at onset & $5.49(2.99-10.06)$ \\
\hline Symptomatic epilepsy & $3.42(2.21-5.27)$ \\
\hline Abnormal EEG & $2.08(1.16-3.74)$ \\
\hline Febrile seizure & $1.31(1.02-1.68)$ \\
\hline $\begin{array}{l}\text { Abnormal neurologic } \\
\text { image }\end{array}$ & $2.78(1.91-4.05)$ \\
\hline Intellectual disability & $3.38(2.16-5.31)$ \\
\hline Neurologic abnormality & $8.61(2.96-24.99)$ \\
\hline Status epilepticus & $3.30(2.36-4.63)$ \\
\hline Psychiatric comorbidities & $1.93(1.60-2.33)$ \\
\hline
\end{tabular}

Note: Adapted from Kalilani L, Sun X, Pelgrims B, Noack-Rink M, Villanueva V. The epidemiology of drug-resistant epilepsy: a systematic review and meta-analysis. Epilepsia. 2018;59(12):2179/-2193. doi:10.1 II I/epi. $14596 .{ }^{18}$

As shown in Table 1, several factors are associated with the risk of evolution to DRE in patients with newly diagnosed epilepsy. Although a lot of variations in the definitions of the risk factors across studies limit the interpretation of available epidemiological data, the factors with the strongest association with DRE are epilepsy onset in the infancy, intellectual disability, symptomatic epilepsy and abnormal neurological exam. ${ }^{18}$ Although number of seizures before treatment initiation has been suggested to be a risk factor for DRE, delaying treatment initiation in patients who have presented one or two seizures does not modify the probability of achieving seizure freedom. ${ }^{19}$ Better identification of patients at high risk of DRE at treatment initiation remains an important challenge. A machine learning approach identified patients at high risk of DRE two years earlier than the current practice. ${ }^{20}$ However, patterns of evolution vary among patients, with in some patients DRE present at onset; ${ }^{3}$ but other experience a relapse-remitting pattern. $^{2}$

DRE can occur in all types of epilepsy syndromes, though the risk differs across them. In particular, although idiopathic generalized epilepsies are often considered as drug responsive, the long-term remission rates ranges from $64 \%$ to $82 \%$ of treated patients. ${ }^{21,22}$ Long-term follow-up data thus shows that the proportion of patients who suffer from seizures despite ASD, even at low annual rate, is almost similar as the proportion of drug-resistance in adult focal epilepsy. ${ }^{23,24}$ Poor outcome factors for idiopathic generalized epilepsies were recently evaluated ${ }^{25}$ and were early seizure onset (before 13 years), multiple types of generalized seizures, history of status epilepticus, EEG with epileptiform activity (mainly polyspikes) and side effects of ASD. Resistance to sodium valproate was found to be the most important prognostic factor for refractory seizures ${ }^{26}$ and suggest that "drug resistant idiopathic generalized epilepsies" may not be considered unless patients have been treated with sodium valproate.

In patients with epilepsy related to a genetic etiology, drug-resistance is particularly frequent, especially in those with severe developmental and epileptic encephalopathies. For instance, drug-resistance is virtually observed in all patients with Dravet Syndrome. ${ }^{27,28}$ However, SCN1A mutations, which are associated with Dravet syndrome, can also be associated with Genetic Epilepsy with Febrile Seizures Plus (GEFS + ) ${ }^{29}$ resulting in potential implications for treatment specially to avoid pseudoresistance. In addition, some Single Nucleotide Polymorphisms (SNPs) of the intronic regions of the SCN1A genes are significantly associated with drug resistance, ${ }^{30}$ thus emerging as potential risk factors for drug resistance.

Concerning adult focal epilepsies, a great variability exists across the underlying etiologies. A diagnosis in the second decade of life, with more than one seizure with impaired awareness per month, mesial temporal sclerosis and bitemporal epilepsy are risk factors for DRE in focal epilepsy. ${ }^{31}$ The drug resistance risk is higher in patients with cortical dysgenesis or hippocampal atrophy. ${ }^{32}$

\section{Pharmacological Management Alternative Monotherapy or Adjunctive Therapy?}

Although polytherapy is frequently used in patients with DRE, few evidences support a significant benefit of adjunctive therapy in comparison with alternative monotherapy. It should however be reminded that all ASDs are first evaluated as adjunctive therapy in Phase III studies conducted in patients with DRE. ${ }^{33}$ Accordingly, the level of evidence about the efficacy of available ASDs in the add-on therapy in DRE is high. Rare studies compared efficacy of alternative monotherapy versus adjunctive therapy in patients who had not responded to ASD. In two 
studies conducted in Italy, no difference was observed between the two arms. ${ }^{34,35}$ However, a large majority of patients included in these studies failed only one monotherapy, and therefore did not fulfil criteria of DRE. Patients were randomized in one study, whereas the other was observational. However, because the randomized study needed to be pragmatic and adapted to clinical practice, no blinding procedure was used. Most importantly, the seizure freedom rate was very different between the two studies, suggesting different patient populations. While remission was observed whatever the treatment strategy in $72 \%$ of patients in one study, ${ }^{35}$ the 12 -month probability of remaining seizure-free was about $15 \%$ in the other. $^{34}$

On the other hand, the benefit of polytherapy in comparison with monotherapy has been indirectly shown in other studies, especially when one considers the positive impact of the association of lamotrigine and sodium valproate in patients with $\mathrm{DRE}^{36}$ or of clobazam with stiripentol of cannabidiol in Dravet Syndrome. ${ }^{37,38}$

However, there are also a large amount of data demonstrating the consequences of ASD-related adverse events on patients' quality of life. ${ }^{39}$ In patients with DRE, it has been shown that the negative impact on daily quality of life of ASD-related adverse events is greater than seizure frequency, ${ }^{40}$ especially when ASDs alter cognitive functioning, mood or coordination. ${ }^{41}$ In this context, reducing ASD load might be beneficial in some patients, though seizure freedom is not achieved.

Overall, the choice between monotherapy and polytherapy should probably vary across patients, depending on types of seizure, seizure frequency, underlying epileptic syndrome and ASD tolerability.

\section{Choosing ASD Upon Its Efficacy Data in DRE?}

As discussed above, one of the main principles is to use ASDs adapted to the patient's epilepsy syndrome. All licensed ASDs, but ethosuximide, stiripentol and cannabidiol, can be used in drug-resistant focal epilepsy. In idiopathic generalized epilepsy as well as in some developmental epileptic encephalopathies, ${ }^{42}$ some ASDs should be used with caution, whereas broad-spectrum medications, such as levetiracetam, sodium valproate, topiramate, perampanel, barbiturates or zonisamide, may be preferred.
Beyond this principle, basing the choice of ASD on a hierarchy of efficacy between them is difficult to achieve, both in primary generalized epilepsy ${ }^{43}$ and in focal epilepsies. ${ }^{44}$ In the absence of head-to-head trials comparing ASDs in DRE, efficacy evaluation relies on indirect comparisons of phase III randomized placebocontrolled trials. Using this approach, no significant difference in efficacy was observed between ASD available in drug-resistant focal epilepsy ${ }^{44}$ or in primary generalized epilepsy. ${ }^{43}$ Another methodological approach is to compare data of the same randomized studies using network meta-analyses. ${ }^{45}$ However, results were similar and mostly showed no statistically significant differences between ASDs for adjunctive therapy. ${ }^{45}$ However, all these systematic reviews suffered from similar limitations. ${ }^{44,45}$ Because all ASDs are compared to placebo, a key issue is to assume that response to placebo is similar across studies. However, as demonstrated in other diseases, this proved to be wrong. In epilepsy, it has been shown that response to placebo is greater in children than in adults ${ }^{46}$ and, in the latter, has progressively increased over the last 20 years. $^{44}$ The reasons underlying this evolution are unclear but they are probably multifactorial, including variation in the regression to the mean effect, placebo effect and Hawthorne effect. ${ }^{44}$ Whether or not the evolution of how the studies are coordinated, with increased number of study sites and involvement of a very large number of countries in a single trial, has also played a role remains to be determined. However, the validity of comparing placebo-controlled studies performed in the $90 \mathrm{~s}$ with studies performed more recently is highly debatable. ${ }^{44}$

Another important issue is the primary outcome of the phase III randomized placebo-controlled trials and therefore of their meta-analyses. According to the FDA and the EMA guidelines, the primary outcome of these trials is the decrease of the median monthly seizure frequency or the $50 \%$ responder rate (ie, the number of patients who achieve $50 \%$ decrease in seizure frequency during the treatment period in comparison with the baseline period), respectively. In contrast, the evaluation of the seizure freedom rate is rarely informative because of methodological issues ${ }^{47}$ and because patients included in these studies typically do not achieve period of seizure remission. Accordingly, no difference in seizure freedom rate is usually observed between ASD and placebo. ${ }^{44}$ It should however be noted that a recent study evaluating a new ASD, Cenobamate, reported that $21 \%$ of patients allocated 
to the $400 \mathrm{mg}$ arm were seizure free in comparison with $1 \%$ in the placebo group. ${ }^{48}$ Although a patient can suffer from different seizure types, the data of phase III randomized placebo-controlled trials are usually informative for only one of them, corresponding to the primary outcome. For instance, patients with drug-resistant focal epilepsy can present focal seizures and focal to bilateral tonicclonic seizures. However, phase III studies are not designed to evaluate focal to bilateral tonic-clonic seizures. ${ }^{49}$ Similarly, studies evaluating ASD in drugresistant idiopathic generalized epilepsies are typically designed to evaluate tonic-clonic seizures but are less informative for other seizure types, including absence or myoclonic seizures. The same issue has also been raised in epileptic encephalopathies, such as Lennox-Gastaut syndrome. $^{50}$

\section{Improving Antiseizure Efficacy with Drugs Acting on Disease-Specific Pharmacological Targets?}

The primary mechanism of action of the drugs currently available in the treatment of epilepsy can be grouped into five broad categories: ${ }^{51}$ (i) Modulation of voltage-gated ion channels, (ii) modulation of neurotransmitter release, (iii) potentiation of GABAergic transmission, (iv) blockade of glutamatergic transmission, and (v) ASD with a different mechanism of action. Apart from few exceptions discussed below, these mechanisms of action share a similar effect on the epileptic networks: through modification of the excitation/inhibition balance, they decrease the excitability of the neuronal network and consequently the risk of seizure initiation or propagation. In contrast, they do not alter the pathophysiological process involved in the epileptogenesis. Accordingly, they can lead to seizure control, but none has showed an antiepileptogenic efficacy $^{52}$ and can be considered as a curative treatment of epilepsy.

True disease-specific treatments remain therefore very rare in DRE. A recent review $^{53}$ presented patient and etiology-adapted treatments. This approach is attractive but relies on identification of a specific etiology mainly genetic and/or metabolic. Inhibition of the mTOR pathway for the treatment of seizures in Tuberous Sclerosis Complex (TSC) could be considered as the prototypic example. Mutations of TSC1 or TSC2 genes are detected in more than $95 \%$ of patients and cause suppression of mTOR (mechanistic target of rapamycin) inhibition, producing an excessive activation of the mTOR signaling pathway and several abnormalities in cell cycle regulation. Beyond decreasing the volume of TSC-related tumors, especially giant astrocytoma or angiomyolipoma, everolimus, a drug inhibiting the mTOR pathway, significantly reduces seizure frequency in patients with TSC. ${ }^{54}$ Because of the role of abnormalities in the mTOR pathways of some focal cortical dysplasia, ${ }^{55,56}$ antiseizure efficacy of everolimus might not be restricted to tuberous sclerosis, ${ }^{57}$ but clinical data are lacking. ${ }^{58}$ Few other examples of disease-specific treatments can be discussed. In patients with GLUT1 deficiency, ketogenic diet represents the standard choice because it provides ketone bodies for brain energy metabolism and thus compensates the brain energy failure syndrome caused by impaired glucose transport across brain tissue barriers. ${ }^{59(\mathrm{p} 1)}$ Fenfluramine, a serotonin agonist acting on $5 \mathrm{HT}_{2 \mathrm{~B} / \mathrm{C}}$ receptor, has recently been shown to have a strong antiseizure efficacy in Dravet syndrome, ${ }^{60}$ in which preclinical data suggesting involvement of serotonin dysfunction in the pathogenesis of epilepsy in this syndrome. ${ }^{61-63}$ In the future, other similar gene-specific therapy might be developed, such as the glutamatergic drug memantine in patients with mutation of GRIN2A gene ${ }^{64}$ or drug acting on voltage-gated potassium channels, including Retigabine, in patients with severe epileptic encephalopathies due to mutation of KCNQ genes. ${ }^{65}$

In addition to these few true disease-specific treatments, it has been suggested that some molecules might be used to target epilepsy-related pathological process that might participate to drug-resistance. Drug-resistance might partly be related to overexpression of $\mathrm{ABC}$ transporters, especially P-glycoprotein. ${ }^{66}$ Verapamil being a competitive inhibitor of $\mathrm{ABC}$ transporters, ${ }^{67}$ its efficacy an adjunctive treatment to ASD has been evaluated in an open-label pilot study. ${ }^{68}$ However, controlled studies are required to formally evaluate its efficacy. The potential interest of diuretics in the treatment of seizures was raised several years ago. ${ }^{69}$ It has been reported very often over the last twenty years that during the period of brain development and during epileptogenesis the inhibitory action of GABAergic transmission could be reversed towards an excitatory action. ${ }^{70}$ This reversal of the effect of GABAergic transmission would depend on the intracellular accumulation of chlorine linked to a modification of the expression of certain membrane transporters of the latter. ${ }^{70}$ Thus, whereas in the adult brain the regulation of chlorine is mainly ensured by the KCC2 transporter, which maintains a low intracellular chlorine 
concentration and thus a hyperpolarizing GABAergic transmission, the situation is different in the immature brain and in immature $^{71}$ and adult ${ }^{72}$ epileptic networks. Indeed, the sodium/potassium/chlorine exchanger NKCC1 is expressed in a pre-dominant manner, favoring the accumulation of intracellular chlorine and consequently modifying the effect of GABAergic transmission towards a depolarizing, potentially pro-epileptogenic action. ${ }^{70}$ Because of its antagonistic action on NKCC1, bumetanide could be a pathway for regulating pathological network activity. Despite encouraging animal studies, ${ }^{73}$ a therapeutic trial in the management of refractory seizures in neonates failed to show an antiseizure efficacy of bumetanide. ${ }^{74}$ However, other NKCC1 antagonists might be developed in the future. ${ }^{75}$ Numerous data have shown the importance of the inflammatory reaction during the epileptogenic process. ${ }^{76}$ Accordingly, drugs with antiinflammatory properties and/or targeting the immune system might be of interest in DRE. Blocking the interleukin 1- $\beta$ pathway has thus been proposed has thus been proposed, using an antagonist of the caspase 1, VX765. Despite interesting data in rodents ${ }^{77}$ and in a phase 2 a study in patients, ${ }^{78}$ the phase $2 \mathrm{~b}$ trial was stopped because of lack of efficacy. It should however be noted that in several situations, including DRE associated with limbic encephalitis. ${ }^{79}$ New Onset Refractory Status Epilepticus (NORSE) or febrile infectionrelated epilepsy syndrome, ${ }^{80}$ anti-inflammatory and/or immunosuppressive drugs must be considered.

\section{Choosing ASD Upon Their Safety Profile?} It is clear that the factors underlying the ASD choice cannot be limited to efficacy data alone. Safety of ASD can be influenced by several factors: (i) patient's age; (ii) patient's gender; (iii) risk of drug-drug interactions, between ASDs and/or with non-ASD molecules; and (iv) patients' comorbidities.

Risk of drug-related adverse effects varies across patients' age. In children, a specific attention needs to be paid to the impact of ASD on cognitive functioning. Some ASD, including sodium valproate, can aggravate attention deficit, ${ }^{81}$ which is very frequent in $\mathrm{DRE}^{82}$ and associated a greater risk of academic difficulties. ${ }^{81}$ Nevertheless, this parameter must also be considered in adults, with particular attention to the elderly. In the later, ASD tolerability might be lower than in young adults because of decrease in drug clearance ${ }^{83}$ and/or of greater susceptibility to nonspecific central nervous system side effects. The risk of fall and injury is thus greater, especially with ASD associated with increased risk of imbalance, such as sodium- channel blockers, ${ }^{84}$ or of impaired alertness, including benzodiazepines. $^{85}$

Another important factor to consider in the elderly is drug-drug interactions, which often lead to the avoidance of enzyme inducers. This issue of drug interaction should also be anticipated regardless of age in the setting of a tumor-related epilepsy where the use of an enzymeinducing ASD may result in a decrease in the efficacy of the chemotherapy or in women in childbearing age with oral contraception. Furthermore, the long-term association between enzyme inducers and atherosclerosis and risk of cardiovascular events might result in considering limiting the use of enzyme inducers in most patients with DRE, especially in those with additional cardiovascular risk factors.

Anticipating the safety issues related to the risk of malformations and/or cognitive deficit in children after inutero exposition to ASD is a key rule in women of childbearing age with new-onset epilepsy. ${ }^{86}$ This aspect remains mandatory in patients with DRE. Polytherapy has traditionally been considered to be associated with a higher risk of major congenital malformations than monotherapy. However, it has been shown the risks are primarily driven by the type of ASD included as polytherapy than the number of ASDs. ${ }^{86}$ As a matter of fact, combination of sodium of valproate or topiramate is an independent risk factor of major congenital malformations. $^{87}$

Lastly, patients' comorbidities must carefully be screened. Without going into an exhaustive list, some frequent examples can be highlighted. Psychiatric comorbidity, either depressive or anxious, is frequent in patients with DRE. ${ }^{88}$ Several drugs are associated with a greater risk of psychiatric complications, including levetiracetam, topiramate, zonisamide or perampanel, ${ }^{89,90}$ and should be used with cautious in these patients. In contrast, other drugs, such as carbamazepine, lamotrigine or sodium valproate, are approved in the treatment of mood disorders. Some treatments are associated with weight gain and should be avoided in overweight patients. Conversely, others, such as topiramate and zonisamide, are associated with weight loss and should be used with caution in patients with eating disorders. Lamotrigine may cause sleep disturbances or be associated with headache, which is important in the context of frequent migraine comorbidity. 


\section{Using Efficacy and Safety Data to Choose ASD Upon a Rational Polytherapy Approach?}

Rational polytherapy has frequently been advocated. It is typically viewed as a way to optimize the efficacy of the association of two ASDs through the complementarity of their mechanisms of action. ${ }^{91,92}$ However, as discussed below, this concept might primarily apply to the choice of the most appropriate balance between the expected benefit of the ASDs combination on seizure frequency and the safety profile of the later.

In theory, three situations can be encountered when two drugs are associated: (i) Additive effect, the effect of the association being the addition of each drug apart; (ii) an antagonist or infra-additive effect; or (iii) a synergistic effect corresponding to a supra-additive effect. Synergistic association is the most interesting, but the majority of preclinical and clinical studies are not designed to distinguish synergic, additive or antagonist associations. ${ }^{51}$

A systematic review ${ }^{93}$ evaluated published preclinical data with a possible synergistic effect of the association of ASD. A total of 107 studies were included, with a synergistic effect reported in 54\%. However, only 65 studies had appropriate methods and only 27 had considered pharmacokinetic intracerebral variations. Accordingly, the majority of preclinical studies were not performed to distinguish synergistic, additive, and antagonistic activities of ASD. ${ }^{93,94}$ When restricted to studies with appropriate methods, as many synergic and antagonistic associations were observed, without correlation with the mechanisms of the ASD. ${ }^{93}$

Similarly, no clinical data formally support a synergistic association between ASD. However, rare exceptions must be noticed. The most important is the association of lamotrigine and sodium valproate which proved to be better that combination of lamotrigine with carbamazepine or phenytoin. The value of this association was since reported in other studies. ${ }^{95}$ As discussed above, the other exceptions have been reported in Dravet syndrome with association of stiripentol with sodium valproate and clobazam ${ }^{37}$ or with association of cannabidiol and clobazam. ${ }^{38}$

However, a key aspect of rational polytherapy is that it should not be only viewed as a way to improve efficacy but also as an important factor for ASD tolerability. ${ }^{51}$ Associations of ASDs can favor the occurrence of adverse events. $^{92}$ This is particularly true for sodium channel blockers which associations can increase the risk of vertigo, ataxia, diplopia. In a pooled analysis of lacosamide data, the adverse event rate doubled when this ASD was associated another sodium channel blocker compared with association with an ASD with other mechanism of action. ${ }^{96}$ Similar observations were reported for association of carbamazepine with lamotrigine. ${ }^{97}$

While in newly diagnosed epilepsy, quality of life primarily associated with complete seizure control, the quality of life of patients suffering from DRE is primarily driven by the occurrence of ASD-related adverse events, ${ }^{40}$ especially those affecting mood, cognition and coordination. ${ }^{41}$ Anticipating the risk of occurrence of ASD-related adverse events is therefore of paramount importance. Accordingly, limiting the association of ASD with the same mechanism of action may be important. ${ }^{51}$

More generally, in patients who have failed several ASDs, the decision to substitute ASD regimen to another should always be individualized in order to balance at the patient individual level the expected efficacy of the new ASD and its safety profile. Accordingly, a combination might be useful in a given patient but not pertinent in another because the risk of adverse events resulting from her/his comorbidities overcomes the potential benefit of that combination. Maintaining the same ASD regimen despite persistent seizures might thus be the best options in certain patients. However, the benefit in terms of efficacy might vary across seizure types. In patients with frequent tonic-clonic seizures, the risks of seizure-related complications, including the risk of SUDEP, ${ }^{9}$ might justify pursuing active treatment revision.

\section{Non-Pharmacological Management}

Every patient with DRE should be referred to a reference tertiary center, especially in the perspective of discussing eligibility for epilepsy surgery or neurostimulation. Despite accumulating evidence about the efficacy of epilepsy surgery and the release of specific guidelines, ${ }^{98}$ delay before referring to a tertiary center seems to remain stable, around 15-20 years in North America and Europe $^{99}$

\section{Epilepsy Surgery}

Epilepsy surgery should primarily be considered in patients with drug-resistant focal epilepsy. ${ }^{100}$ There are class I evidences showing superiority of epilepsy surgery over medical management in adults with drug-resistant temporal lobe epilepsy ${ }^{101,102}$ and whatever the localization 
in children with drug-resistant focal epilepsy. ${ }^{103}$ In addition, successful surgery for epilepsy due to early brain lesions despite generalized EEG has been reported in children. ${ }^{104}$

The presurgical evaluation aims to delineate the epileptogenic zone, a benefit/risk balance between resection, disconnection or destruction of a brain region and the minimum neurological deficit. ${ }^{100}$ The minimal examination should include interictal scalp EEG, a dedicated MRI protocol for epilepsy, neuropsychological assessment, completed if required by functional neuroimaging. ${ }^{100}$ Long-term video-EEG to capture seizures is strongly recommended. ${ }^{100}$ Functional MRI is increasingly used to lateralize language and has mainly replaced Wada test in temporal lobe epilepsy. ${ }^{105}$ When data at the end of this first phase are not strong enough to delineate the brain region that needs to be resected or if this latter involves eloquent cortex, invasive video-EEG recordings with intracranial electrodes, strips or grids are used. ${ }^{106}$ Stereoelectro-encephalography is now the principal method for intracranial EEG monitoring in the majority of epilepsy surgery centers in Europe and in the US. ${ }^{107}$

Overall, seizure freedom following epilepsy surgery is achieved by $60-80 \%$ in patients with temporal lobe epilepsy and $40-75 \%$ in patients with extra-temporal lobe epilepsy. ${ }^{100}$ Moreover, the benefit on quality of life, cognitive evolution, particularly for children, and psychiatric comorbidities is positive. ${ }^{108-110}$ This global benefit is also stated in more specific populations, including children with autism spectrum disorders ${ }^{111}$ or older adults (more than 60 years). ${ }^{112}$ It should be emphasized that lesion on MRI is not a prerequisite for epilepsy surgery. ${ }^{108}$ In patients with MRI-negative temporal lobe epilepsy, longterm postoperative seizure-free rates vary from $40 \%$ to $60 \% .{ }^{100}$ Similarly, the presence of a focal MRI abnormality has not been significantly associated with outcome in extra-temporal lobe epilepsy. ${ }^{113}$ However, the best results have been reported in focal cortical dysplasia type $2 \mathrm{~B}$, with up to $92 \%$ of patients achieving seizure freedom after a mean follow-up of 4 years, and no difference between MRI-positive and MRI-negative patients. ${ }^{114}$

Magnetic resonance imaging-guided laser interstitial thermal therapy (MR-guided LiTT) is an alternative to cortical resection. Because of its minimal postoperative morbidity, ${ }^{115,116}$ its use is growing in mesial temporal lobe epilepsy. MR-guided LiTT as well as stereo-EEG- guided radiofrequency thermocoagulation might also be useful in patients not eligible for cortical resection including those with periventricular nodular heterotopia or hypothalamic hamartomas. About $50 \%$ of patients with periventricular nodular heterotopia can achieve seizure remission following stereo-EEG-guided radiofrequency thermocoagulation. ${ }^{117}$

\section{Neurostimulation}

Neurostimulation can be separated into two groups, vagus nerve stimulation (VNS) and brain stimulation, including Deep Brain Stimulation (DBS) and Responsive neurostimulation (RNS). These approaches showed similar efficacy. ${ }^{118}$

VNS is frequently proposed in patients not eligible for epilepsy surgery. The probability to achieve seizure freedom is low, ${ }^{119}$ but there is a positive impact on QOL in $50 \%$ of patients. ${ }^{120}$ Adverse events are not rare, with intraoperative abnormal heart rhythm, dysphonia, dysphagia, surgical site infection and sleep apnea. ${ }^{120}$ Initially validated in focal epilepsy, other studies demonstrated interest of VNS in generalized epilepsies, ${ }^{119}$ such as Lennox Gastaut or Dravet syndromes.

DBS proved to decrease seizure frequency in DRE. The highest level of evidence has been reported for stimulation of the anterior nucleus of the thalamus. ${ }^{121}$ Longterm study reported $69 \%$ reduction in seizure frequency at five years, with two-third of $50 \%$ responders. ${ }^{122}$ Other targets, including centromedian thalamus nucleus, may also be associated with antiseizure efficacy, especially in Lennox-Gastaut syndrome. ${ }^{123}$

RNS is a closed-loop cortical stimulation device. Cortical strips and/or depth electrodes deliver customized neurostimulation in the seizure onset zone and prevent seizure propagation based on the electrocorticographic detection of abnormalities. ${ }^{124}$ In a pivotal double-blind sham-controlled parallel-group RCT which included 191 adult patients with focal DRE, RNS system therapy showed showing an overall $29 \%$ responder rate at the end of the double-blind phase and $45 \%$ at 2 years in the open-label extension. ${ }^{125}$

\section{Diets}

Ketogenic diet has proven its usefulness in children and adolescents with DRE. ${ }^{126,127}$ In adults, the use of ketogenic diet remains uncertain and needs further research. In 
case of ketogenic diet, ASD concentrations have to be monitored, due to pharmacokinetic interactions between diet and ASD. ${ }^{128}$ A potential beneficial effect of probiotics in DRE has been assessed by a pilot study. ${ }^{129}$ Thirty percent of patients with the probiotic mixture for four months experienced for a greater than $50 \%$ of seizure reduction, but further investigations are needed.

\section{Comprehensive Care Adaptations}

As mentioned above, the main goals in patients with DRE is to optimize quality of life. QOL determinant in DRE are multifactorial. The relation between seizure frequency and QOL is blurry, some authors showing a statistically significative association, ${ }^{130-132}$ whereas others do not. ${ }^{40}$ Conversely, poor tolerance of ASD has a negative impact on QOL, adverse events of treatment being a strong prognostic variable for QOL. ${ }^{40}$

Furthermore, the existence of a psychiatric comorbidity - anxiety or depression - is independently associated with QOL alteration. ${ }^{40,133,134}$ The burden of epilepsy could also vary according to the type of seizure, given that generalized tonic-clonic seizures, even though less frequent, are associated with more severe seizure-related complications and are associated with increased risk of depression and anxiety. ${ }^{135}$ For children and adolescents, a close relation exists between QOL and cognitive impairment, particularly attention deficit. ${ }^{82,136}$ In adults, specific data are scarce but point to the same direction, ${ }^{137}$ particularly the relation with cognitive impairment and treatment adverse events. $^{41}$ In patients older than 50 with drug resistant temporal epilepsy, an impaired cognitive profile (verbal memory performance) was assessed in half of patients by neuropsychological examinations. ${ }^{138}$

A specific period with higher risk for these complications is the transition between adolescence and adulthood. Patients and their families face several issues, including the change from childhood neurologist to adult neurologist. Over the past years, several studies have emphasized the importance of adequately manage this critical period, especially with dedicated transitions clinics in the most difficult situations. ${ }^{139}$

\section{Psychologic Care}

Epilepsy is associated with 2 or 3 -fold more psychiatric comorbidities than population and about a third of drug resistant patients are affected by these latter. ${ }^{88,140}$ Psychiatric comorbidities include mood disorders, anxiety and some psychotic disorders. Depression prevalence is higher in epileptic population, notably in DRE, and suicide risk is three times higher than the risk in general population. ${ }^{141}$ Anxiety comes next, ${ }^{140}$ with panic disorder, social phobia, obsessive disorder, generalized anxiety. ${ }^{142}$ More than a third of children undergoing epilepsy surgery evaluation reported unmet overall healthcare needs, ${ }^{143}$ with greater risk if the patient presented depressive symptoms or with young or unemployed caregivers. In this context, monitoring and care of psychiatric comorbidities is important, especially using dedicated screening scales validated in epilepsy such as the neurological disorders depression inventory for epilepsy (NDDI-E). ${ }^{144}$ Importantly, if a specific treatment is needed, epilepsy does not represent a contraindication for antidepressants, with a selective serotonin reuptake inhibitor as a first line. ${ }^{145}$ VNS is also approved for refractory depression and might be useful for patients DRE and comorbid depression. ${ }^{146}$ Mindfulness therapy is also associated with greater benefits than short-term psychotherapy in QOL, mood, seizure frequency, and verbal memory. ${ }^{147}$

\section{Cognitive Impairment}

Many factors contribute to cognitive impairments in PWE: epilepsy etiology, seizure type, frequency, age of beginning, medical treatment. Moreover, all cognitive functions can be impact to several degrees, such as attention, language, memory, emotions, gestures, executive functions, logic, visuospatial capacities. Given the increased handicap associated with these cognitive difficulties, particularly for formation or professional projects, their early detection is a fundamental element in the ILAE care. ${ }^{148}$

\section{Education and Professional Impacts}

Because occurrence of a seizure at work may expose to a risk of injury, some professional activities are not compatible with DRE. Conversely, some patients suffer from difficulties to achieve their professional objectives not because of seizures per se but because stigmatization. In order to better accompany the patients, several epilepsy centers have created epileptological and occupational physician combined consultations. In children, an individualized host project allows education in ordinary schools or with auxiliary, or adapted education.

\section{Abbreviations}

$\mathrm{ABC}$ transporter, adenosine triphosphate binding cassette; ASD, antiseizure drug; CRISPR, Clustered Regularly Interspaced Short Palindromic Repeats; DBS, deep brain stimulation; DRE, drug resistant epilepsy; GABA, 
Gamma-aminobutyric acid; GEFS+, Genetic Epilepsy with Febrile Seizures Plus; GGE, genetic generalized epilepsy; ILAE, International League Against Epilepsy; MR-guided LiTT, magnetic resonance imaging-guided laser interstitial thermal therapy; NMDR, N-Methyl-d-aspartic acid receptor; mTOR, Mammalian target of rapamycin; QOL, quality of life; RNS, responsive neurostimulation; SNPs, single nucleotide polymorphisms; SUDEP, sudden unexpected death in epilepsy; TMS, transcranial repetitive stimulation; VNS, vagus nerve stimulation.

\section{Disclosure}

D.G reports has nothing to disclose.

S.R received speaker and/or consulting fees from UCB Pharma, EISAI, GW Pharma, Idiorsia, Livanova, Arvelle Therapeutics.

\section{References}

1. Kwan P, Arzimanoglou A, Berg AT, et al. Definition of drug resistant epilepsy: consensus proposal by the ad hoc Task Force of the ILAE commission on therapeutic strategies: definition of drug resistant epilepsy. Epilepsia. 2009;51(6):1069-1077. doi:10.1111/j.1528-1167.2009.02397.x

2. Brodie MJ, Barry SJE, Bamagous GA, Norrie JD, Kwan P. Patterns of treatment response in newly diagnosed epilepsy. Neurology. 2012;78(20):1548-1554. doi:10.1212/ WNL.0b013e3182563b19

3. Kwan P, Brodie MJ. Early identification of refractory epilepsy. $N \quad$ Engl $J \quad$ Med. 2000;342(5):314-319. doi:10.1056/ NEJM200002033420503

4. Schiller Y, Najjar Y. Quantifying the response to antiepileptic drugs: effect of past treatment history. Neurology. 2008;70 (1):54-65. doi:10.1212/01.wnl.0000286959.22040.6e

5. Chen Z, Brodie MJ, Liew D, Kwan P. Treatment outcomes in patients with newly diagnosed epilepsy treated with established and new antiepileptic drugs: a 30-year longitudinal cohort study. JAMA Neurol. 2018;75(3):279. doi:10.1001/ jamaneurol.2017.3949

6. Callaghan BC, Anand K, Hesdorffer D, Hauser WA, French JA. Likelihood of seizure remission in an adult population with refractory epilepsy. Ann Neurol. 2007;62(4):382-389. doi:10.1002/ana.21166

7. Luciano AL, Shorvon SD. Results of treatment changes in patients with apparently drug-resistant chronic epilepsy. Ann Neurol. 2007;62(4):375-381. doi:10.1002/ana.21064

8. Callaghan B, Schlesinger M, Rodemer W, et al. Remission and relapse in a drug-resistant epilepsy population followed prospectively: drug-resistant epilepsy population. Epilepsia. 2011;52 (3):619-626. doi:10.1111/j.1528-1167.2010.02929.x

9. Ryvlin P, Cucherat M, Rheims S. Risk of sudden unexpected death in epilepsy in patients given adjunctive antiepileptic treatment for refractory seizures: a meta-analysis of placebo-controlled randomised trials. Lancet Neurol. 2011;10 (11):961-968. doi:10.1016/S1474-4422(11)70193-4

10. Choi H, Hayat MJ, Zhang R, et al. Drug-resistant epilepsy in adults: outcome trajectories after failure of two medications. Epilepsia. 2016;57(7):1152-1160. doi:10.1111/epi.13406
11. Zaccara G, Mula M, Ferrò B, et al. Do neurologists agree in diagnosing drug resistance in adults with focal epilepsy? Epilepsia. 2019;60(1):175-183. doi:10.1111/epi.14622

12. O' Rourke G, O' Brien JJ. Identifying the barriers to antiepileptic drug adherence among adults with epilepsy. Seizure. 2017;45:160-168. doi:10.1016/j.seizure.2016.12.006

13. Anzellotti F, Dono F, Evangelista G, et al. Psychogenic non-epileptic seizures and pseudo-refractory epilepsy, a management challenge. Front Neurol. 2020;11:461. doi:10.3389/fneur.2020.00461

14. Thomas P, Valton L, Genton P. Absence and myoclonic status epilepticus precipitated by antiepileptic drugs in idiopathic generalized epilepsy. Brain. 2006;129(5):1281-1292. doi:10.1093/brain/awl047

15. Boylan LS, Labovitz DL, Jackson SC, Starner K, Devinsky O. Auras are frequent in idiopathic generalized epilepsy. Neurology. 2006;67(2):343-345. doi:10.1212/01.wnl.0000225185.37081.97

16. Lombroso CT. Consistent EEG focalities detected in subjects with primary generalized epilepsies monitored for two decades. Epilepsia. 1997;38(7):797-812. doi:10.1111/j.1528-1157.1997.tb01467.x

17. Kellinghaus C, Lüders HO. Frontal lobe epilepsy. Epileptic Disord. 2004;6(4):223-239.

18. Kalilani L, Sun X, Pelgrims B, Noack-Rink M, Villanueva V. The epidemiology of drug-resistant epilepsy: a systematic review and meta-analysis. Epilepsia. 2018;59(12):2179-2193. doi:10.1111/ epi. 14596

19. Marson A, Jacoby A, Johnson A, et al. Immediate versus deferred antiepileptic drug treatment for early epilepsy and single seizures: a randomised controlled trial. Lancet. 2005;365(9476):2007-2013. doi:10.1016/S0140-6736(05)66694-9

20. An S, Malhotra K, Dilley C, et al. Predicting drug-resistant epilepsy - a machine learning approach based on administrative claims data. Epilepsy Behavior. 2018;89:118-125. doi:10.1016/j. yebeh.2018.10.013

21. Mohanraj R, Brodie MJ. Outcomes of newly diagnosed idiopathic generalized epilepsy syndromes in a non-pediatric setting. Acta Neurol Scand. 2007;115(3):204-208. doi:10.1111/j.1600-0404.2006.00791.x

22. Szaflarski JP, Lindsell CJ, Zakaria T, Banks C, Privitera MD. Seizure control in patients with idiopathic generalized epilepsies: EEG determinants of medication response. Epilepsy Behavior. 2010;17(4):525-530. doi:10.1016/j.yebeh.2010.02.005

23. Holtkamp M, Kowski AB, Merkle H, Janz D. Long-term outcome in epilepsy with grand mal on awakening: forty years of follow-up. Ann Neurol. 2014;75(2):298-302. doi:10.1002/ana.24103

24. Senf P, Schmitz B, Holtkamp M, Janz D. Prognosis of juvenile myoclonic epilepsy 45 years after onset: seizure outcome and predictors. Neurology. 2013;81(24):2128-2133. doi:10.1212/01. wnl.0000437303.36064.f8

25. Gomez-Ibañez A, McLachlan RS, Mirsattari SM, Diosy DC, Burneo JG. Prognostic factors in patients with refractory idiopathic generalized epilepsy. Epilepsy Res. 2017;130:69-73. doi:10.1016/j.eplepsyres.2017.01.011

26. Gesche J, Khanevski M, Solberg C, Beier CP. Resistance to valproic acid as predictor of treatment resistance in genetic generalized epilepsies. Epilepsia. 2017;58(4):e64-e69. doi:10.1111/ epi.13702

27. Catarino CB, Liu JYW, Liagkouras I, et al. Dravet syndrome as epileptic encephalopathy: evidence from long-term course and neuropathology. Brain. 2011;134(10):2982-3010. doi:10.1093/ brain/awr129

28. Genton P, Velizarova R, Dravet C. Dravet syndrome: the long-term outcome: long-term outcome. Epilepsia. 2011;52:44-49. doi:10.1111/j.1528-1167.2011.03001.x

29. Singh R, Scheffer IE, Crossland K, Berkovic SF. Generalized epilepsy with febrile seizures plus: a common childhood-onset genetic epilepsy syndrome. Ann Neurol. 1999;45(1):75-81. doi:10.1002/1531-8249(199901)45:1<75::aid-art13>3.0.co;2-w 
30. Margari L, Legrottaglie AR, Vincenti A, et al. Association between SCN1A gene polymorphisms and drug resistant epilepsy in pediatric patients. Seizure. 2018;55:30-35. doi:10.1016/j. seizure.2018.01.002

31. Roy PL, Ronquillo LH, Ladino LD, Tellez-Zenteno JF. Risk factors associated with drug resistant focal epilepsy in adults: a case control study. Seizure. 2019;73:46-50. doi:10.1016/j. seizure.2019.10.020

32. Semah F, Picot M-C, Adam C, et al. Is the underlying cause of epilepsy a major prognostic factor for recurrence? Neurology. 1998;51(5):1256-1262. doi:10.1212/WNL.51.5.1256

33. Perucca E, French J, Bialer M. Development of new antiepileptic drugs: challenges, incentives, and recent advances. Lancet Neurol. 2007;6(9):793-804. doi:10.1016/S1474-4422(07)70215-6

34. Beghi E, Gatti G, Tonini C, et al. Adjunctive therapy versus alternative monotherapy in patients with partial epilepsy failing on a single drug: a multicentre, randomised, pragmatic controlled trial. Epilepsy Res. 2003;57(1):1-13. doi:10.1016/j.eplepsyres.2003.09.007

35. Millul A, Iudice A, Adami M, et al. Alternative monotherapy or add-on therapy in patients with epilepsy whose seizures do not respond to the first monotherapy: an Italian multicenter prospective observational study. Epilepsy Behav. 2013;28(3):494-500. doi:10.1016/j.yebeh.2013.05.038

36. Brodie MJ, Yuen AWC. Lamotrigine substitution study: evidence for synergism with sodium valproate? Epilepsy Res. 1997;26 (3):423-432. doi:10.1016/S0920-1211(96)01007-8

37. Chiron C, Marchand M, Tran A, et al. Stiripentol in severe myoclonic epilepsy in infancy: a randomised placebo-controlled syndrome-dedicated trial. Lancet. 2000;356(9242):1638-1642. doi:10.1016/S0140-6736(00)03157-3

38. Devinsky O, Cross JH, Laux L, et al. Trial of cannabidiol for drug-resistant seizures in the Dravet syndrome. $N$ Engl J Med. 2017;376(21):2011-2020. doi:10.1056/NEJMoa1611618

39. Perucca P, Gilliam FG. Adverse effects of antiepileptic drugs. Lancet Neurol. 2012;11(9):792-802. doi:10.1016/S1474-4422(12)70153-9

40. Gilliam FG, Fessler AJ, Baker G, Vahle V, Carter J, Attarian H. Systematic screening allows reduction of adverse antiepileptic drug effects: a randomized trial. Neurology. 2004;62(1):23-27. doi:10.1212/WNL.62.1.23

41. Perucca P, Carter J, Vahle V, Gilliam FG. Adverse antiepileptic drug effects: toward a clinically and neurobiologically relevant taxonomy. Neurology. 2009;72(14):1223-1229. doi:10.1212/01. wnl.0000345667.45642.61

42. Perucca E, Gram L, Avanzini G, Dulac O. Antiepileptic drugs as a cause of worsening seizures. Epilepsia. 1998;39(1):5-17. doi:10.1111/j.1528-1157.1998.tb01268.x

43. Rheims S, Ryvlin P. Pharmacotherapy for tonic-clonic seizures. Expert Opin Pharmacother. 2014;15(10):1417-1426. doi:10.1517/14656566.2014.915029

44. Rheims S, Perucca E, Cucherat M, Ryvlin P. Factors determining response to antiepileptic drugs in randomized controlled trials. A systematic review and meta-analysis: response to AEDs in Randomized Trials. Epilepsia. 2011. doi:10.1111/j.15281167.2010.02915.x

45. Thieffry S, Klein P, Baulac M, et al. Understanding the challenge of comparative effectiveness research in focal epilepsy: a review of network meta-analyses and real-world evidence on antiepileptic drugs. Epilepsia. 2020;61(4):595-609. doi:10.1111/epi.16476

46. Rheims S, Cucherat M, Arzimanoglou A, Ryvlin P. Greater response to placebo in children than in adults: a systematic review and meta-analysis in drug-resistant partial epilepsy. PLoS Med. 2008;5(8):e166. doi:10.1371/journal.pmed.0050166

47. Gazzola DM, Balcer LJ, French JA. Seizure-free outcome in randomized add-on trials of the new antiepileptic drugs. Epilepsia. 2007;48 (7):1303-1307. doi:10.1111/j.1528-1167.2007.01136.x
48. Krauss GL, Klein P, Brandt C, et al. Safety and efficacy of adjunctive cenobamate (YKP3089) in patients with uncontrolled focal seizures: a multicentre, double-blind, randomised, placebo-controlled, dose-response trial. Lancet Neurol. 2020;19 (1):38-48. doi:10.1016/S1474-4422(19)30399-0

49. Hemery C, Ryvlin P, Rheims S. Prevention of generalized tonic-clonic seizures in refractory focal epilepsy: a meta-analysis. Epilepsia. 2014;55(11):1789-1799. doi:10.1111/ epi. 12765

50. Arzimanoglou A, French J, Blume WT, et al. Lennox-Gastaut syndrome: a consensus approach on diagnosis, assessment, management, and trial methodology. Lancet Neurol. 2009;8(1):82-93. doi:10.1016/S1474-4422(08)70292-8

51. Guery D, Rheims S. Is the mechanism of action of antiseizure drugs a key element in the choice of treatment? Fundam Clin Pharmacol. 2020;fcp.12614. doi:10.1111/fcp.12614

52. Trinka E, Brigo F. Antiepileptogenesis in humans: disappointing clinical evidence and ways to move forward. Curr Opin Neurol. 2014;27(2):227-235. doi:10.1097/ WCO.0000000000000067

53. Perucca P, Perucca E. Identifying mutations in epilepsy genes: impact on treatment selection. Epilepsy Res. 2019;152:18-30. doi:10.1016/j.eplepsyres.2019.03.001

54. French JA, Lawson JA, Yapici Z, et al. Adjunctive everolimus therapy for treatment-resistant focal-onset seizures associated with tuberous sclerosis (EXIST-3): a Phase 3, randomised, double-blind, placebo-controlled study. Lancet. 2016;388 (10056):2153-2163. doi:10.1016/S0140-6736(16)31419-2

55. Mirzaa GM, Campbell CD, Solovieff N, et al. Association of MTOR mutations with developmental brain disorders, including megalencephaly, focal cortical dysplasia, and pigmentary mosaicism. JAMA Neurol. 2016;73(7):836. doi:10.1001/ jamaneurol.2016.0363

56. Baldassari S, Ribierre T, Marsan E, et al. Dissecting the genetic basis of focal cortical dysplasia: a large cohort study. Acta Neuropathol. 2019;138(6):885-900. doi:10.1007/s00401-019-02061-5

57. Griffith JL, Wong M. The mTOR pathway in treatment of epilepsy: a clinical update. Future Neurol. 2018;13(2):49-58. doi:10.2217/fnl-2018-0001

58. Guerrini R, Duchowny M, Jayakar P, et al. Diagnostic methods and treatment options for focal cortical dysplasia. Epilepsia. 2015;56(11):1669-1686. doi:10.1111/epi.13200

59. Klepper J, Akman C, Armeno M, et al. Glut1 Deficiency Syndrome (Glut1DS): state of the art in 2020 and recommendations of the international Glut1DS study group. Epilepsia Open. 2020;5(3):354-365. doi:10.1002/epi4.12414

60. Lagae L, Sullivan J, Knupp K, et al. Fenfluramine hydrochloride for the treatment of seizures in Dravet syndrome: a randomised, double-blind, placebo-controlled trial. Lancet. 2019;394 (10216):2243-2254. doi:10.1016/S0140-6736(19)32500-0

61. Hatini PG, Commons KG. Serotonin abnormalities in Dravet syndrome mice before and after the age of seizure onset. Brain Res. 2019;1724:146399. doi:10.1016/j.brainres.2019.146399

62. Griffin AL, Jaishankar P, Grandjean J-M, Olson SH, Renslo AR, Baraban SC. Zebrafish studies identify serotonin receptors mediating antiepileptic activity in Dravet syndrome. Brain Commun. 2019;1(1):fcz008. doi:10.1093/braincomms/fcz008

63. Griffin A, Hamling KR, Knupp K, Hong S, Lee LP, Baraban SC. Clemizole and modulators of serotonin signalling suppress seizures in Dravet syndrome. Brain. 2017;aww342. doi:10.1093/ brain/aww342

64. Pierson TM, Yuan H, Marsh ED, et al. GRIN2A mutation and early-onset epileptic encephalopathy: personalized therapy with memantine. Ann Clin Transl Neurol. 2014;1(3):190-198. doi:10.1002/acn3.39 
65. Ihara Y, Tomonoh Y, Deshimaru M, et al. Retigabine, a Kv7.2/ Kv7.3-channel opener, attenuates drug-induced seizures in knock-in mice harboring Kcnq2 Mutations. PLoS One. 2016;11 (2):e0150095. doi:10.1371/journal.pone.0150095

66. Löscher W, Potschka H. Drug resistance in brain diseases and the role of drug efflux transporters. Nat Rev Neurosci. 2005;6 (8):591-602. doi:10.1038/nrn1728

67. Leandro K, Bicker J, Alves G, Falcão A, Fortuna A. ABC transporters in drug-resistant epilepsy: mechanisms of upregulation and therapeutic approaches. Pharmacol Res. 2019;144:357-376. doi:10.1016/j. phrs.2019.04.031

68. Asadi-Pooya AA, Razavizadegan SMA, Abdi-Ardekani A, Sperling MR. Adjunctive use of verapamil in patients with refractory temporal lobe epilepsy: a pilot study. Epilepsy Behavior. 2013;29(1):150-154. doi:10.1016/j.yebeh.2013.07.006

69. Hesdorffer DC, Stables JP, Hauser WA, Annegers JF, Cascino G. Are certain diuretics also anticonvulsants? Ann Neurol. 2001;50 (4):458-462. doi:10.1002/ana.1136

70. Ben-Ari Y, Gaiarsa J-L, Tyzio R, Khazipov R. GABA: a pioneer transmitter that excites immature neurons and generates primitive oscillations. Physiol Rev. 2007;87(4):1215-1284. doi:10.1152/ physrev.00017.2006

71. Khalilov I, Holmes GL, Ben-Ari Y. In vitro formation of a secondary epileptogenic mirror focus by interhippocampal propagation of seizures. Nat Neurosci. 2003;6(10):1079-1085. doi: $10.1038 / \mathrm{nn} 1125$

72. Cohen I. On the origin of interictal activity in human temporal lobe epilepsy in vitro. Science. 2002;298(5597):1418-1421. doi:10.1126/science. 1076510

73. Dzhala VI, Talos DM, Sdrulla DA, et al. NKCC1 transporter facilitates seizures in the developing brain. Nat Med. 2005;11 (11):1205-1213. doi:10.1038/nm1301

74. Pressler RM, Boylan GB, Marlow N, et al. Bumetanide for neonatal seizures-back from the cotside. Nat Rev Neurol. 2015;11(12):724. doi:10.1038/nrneurol.2015.116

75. Hampel P, Römermann K, Gramer M, Löscher W. The search for brain-permeant NKCC1 inhibitors for the treatment of seizures: pharmacokinetic-pharmacodynamic modelling of NKCC1 inhibition by azosemide, torasemide, and bumetanide in mouse brain. Epilepsy Behav. 2021;114(PtA):107616. doi:10.1016/j. yebeh.2020.107616

76. Vezzani A, French J, Bartfai T, Baram TZ. The role of inflammation in epilepsy. Nat Rev Neurol. 2011;7(1):31-40. doi:10.1038/ nrneurol.2010.178

77. Ravizza T, Lucas S-M, Balosso S, et al. Inactivation of caspase-1 in rodent brain: a novel anticonvulsive strategy. Epilepsia. 2006;47(7):1160-1168. doi:10.1111/j.15281167.2006.00590.x

78. Bialer M, Johannessen SI, Levy RH, Perucca E, Tomson T, White HS. Progress report on new antiepileptic drugs: a summary of the Eleventh Eilat Conference (EILAT XI). Epilepsy Res. 2013;103(1):2-30. doi:10.1016/j. eplepsyres.2012.10.001

79. Vogrig A, Joubert B, André-Obadia N, Gigli GL, Rheims S, Honnorat J. Seizure specificities in patients with antibody-mediated autoimmune encephalitis. Epilepsia. 2019;60 (8):1508-1525. doi:10.1111/epi.16282

80. Hirsch LJ, Gaspard N, van Baalen A, et al. Proposed consensus definitions for new-onset refractory status epilepticus (NORSE), febrile infection-related epilepsy syndrome (FIRES), and related conditions. Epilepsia. 2018;59(4):739-744. doi:10.1111/ epi. 14016

81. Rheims S, Auvin S. Attention deficit/hyperactivity disorder and epilepsy. Curr Opin Neurol. 2021;34(2):219-225. doi:10.1097/ WCO.0000000000000903
82. Sherman EMS, Slick DJ, Connolly MB, Eyrl KL. ADHD, Neurological correlates and health-related quality of life in severe pediatric epilepsy. Epilepsia. 2007;48(6):1083-1091. doi:10.1111/ j.1528-1167.2007.01028.x

83. Perucca E. Clinical pharmacokinetics of new-generation antiepileptic drugs at the extremes of age. Clin Pharmacokinet. 2006;45 (4):351-363. doi:10.2165/00003088-200645040-00002

84. Sirven JI, Fife TD, Wingerchuk DM, Drazkowski JF. Secondgeneration antiepileptic drugs' impact on balance: a metaanalysis. Mayo Clin Proc. 2007;82(1):40-47. doi:10.4065/82.1.40

85. Finkle WD, Der JS, Greenland S, et al. Risk of fractures requiring hospitalization after an initial prescription for zolpidem, alprazolam, lorazepam, or diazepam in older adults. J Am Geriatr Soc. 2011;59(10):1883-1890. doi:10.1111/j.1532-5415.2011.03591.x

86. Tomson T, Battino D, Bromley R, et al. Management of epilepsy in pregnancy: a report from the International league against epilepsy task force on women and pregnancy. Epileptic Disord. 2019;21(6):497-517. doi:10.1684/epd.2019.1105

87. Keni RR, Jose M, Sarma PS, Thomas SV. For the Kerala Registry of Epilepsy and Pregnancy Study Group. Teratogenicity of antiepileptic dual therapy: dose-dependent, drug-specific, or both? Neurology. 2018;90(9):e790-e796. doi:10.1212/WNL.0000000000005031

88. Kanner AM. Management of psychiatric and neurological comorbidities in epilepsy. Nat Rev Neurol. 2016;12(2):106-116. doi:10.1038/nrneurol.2015.243

89. Chen Z, Lusicic A, O’Brien TJ, Velakoulis D, Adams SJ, Kwan P. Psychotic disorders induced by antiepileptic drugs in people with epilepsy. Brain. 2016;139(10):2668-2678. doi:10.1093/brain/ aww196

90. Mula M. The pharmacological management of psychiatric comorbidities in patients with epilepsy. Pharmacol Res. 2016;107:147-153. doi:10.1016/j.phrs.2016.03.022

91. Verrotti A, Tambucci R, Di Francesco L, et al. The role of polytherapy in the management of epilepsy: suggestions for rational antiepileptic drug selection. Expert Rev Neurother. 2020;20(2):167-173. doi:10.1080/14737175.2020.1707668

92. Stephen LJ, Forsyth M, Kelly K, Brodie MJ. Antiepileptic drug combinations-Have newer agents altered clinical outcomes? Epilepsy Res. 2012;98(2-3):194-198. doi:10.1016/j. eplepsyres.2011.09.008

93. Jonker DM, Voskuyl RA, Danhof M. Synergistic Combinations of Anticonvulsant Agents: what Is the Evidence from Animal Experiments? Epilepsia. 2007;48(3):412-434. doi:10.1111/ j.1528-1167.2006.00952.x

94. Stafstrom CE. Mechanisms of action of antiepileptic drugs: the search for synergy. Curr Opin Neurol. 2010;23(2):157-163. doi:10.1097/WCO.0b013e32833735b5

95. Poolos NP, Warner LN, Humphreys SZ, Williams S. Comparative efficacy of combination drug therapy in refractory epilepsy. Neurology. 2012;78(1):62-68. doi:10.1212/ WNL.0b013e31823ed0dd

96. Sake J-K, Hebert D, Isojärvi J, et al. A pooled analysis of lacosamide clinical trial data grouped by mechanism of action of concomitant antiepileptic drugs. CNS Drugs. 2010;24 (12):1055-1068. doi:10.2165/11587550-000000000-00000

97. Besag FMC, Berry DJ, Pool F, Newbery -J-JE, Subel B. Carbamazepine Toxicity with Lamotrigine: pharmacokinetic or Pharmacodynamic Interaction? Epilepsia. 1998;39(2):183-187. doi:10.1111/j.1528-1157.1998.tb01356.x

98. Engel J, Wiebe S, French J, et al. Practice parameter: temporal lobe and localized neocortical resections for epilepsy: report of the Quality Standards Subcommittee of the American Academy of Neurology, in Association with the American Epilepsy Society and the American Association of Neurological Surgeons. Neurology. 2003;60(4):538-547. doi:10.1212/01. WNL.0000055086.35806.2D 
99. Haneef Z, Stern J, Dewar S, Engel J. Referral pattern for epilepsy surgery after evidence-based recommendations: a retrospective study. Neurology. 2010;75(8):699-704. doi:10.1212/ WNL.0b013e3181 eee457

100. Ryvlin P, Cross JH, Rheims S. Epilepsy surgery in children and adults. Lancet Neurol. 2014;13(11):1114-1126. doi:10.1016/ S1474-4422(14)70156-5

101. Wiebe S, Blume WT, Girvin JP, Eliasziw M. A randomized, controlled trial of surgery for temporal-lobe epilepsy. $N$ Engl J Med. 2001;345 (5):311-318. doi:10.1056/NEJM200108023450501

102. Engel J. Early surgical therapy for drug-resistant temporal lobe epilepsy: a randomized trial. JAMA. 2012;307(9):922. doi:10.1001/jama.2012.220

103. Dwivedi R, Ramanujam B, Chandra PS, et al. Surgery for drug-resistant epilepsy in children. $N$ Engl J Med. 2017;377 (17):1639-1647. doi:10.1056/NEJMoa1615335

104. Wyllie E, Lachhwani DK, Gupta A, et al. Successful surgery for epilepsy due to early brain lesions despite generalized EEG findings. Neurology. 2007;69(4):389-397. doi:10.1212/01. wnl.0000266386.55715.3f

105. Janecek JK, Swanson SJ, Sabsevitz DS, et al. Language lateralization by fMRI and Wada testing in 229 patients with epilepsy: rates and predictors of discordance. Epilepsia. 2013;54 (2):314-322. doi:10.1111/epi.12068

106. Thijs RD, Surges R, O'Brien TJ, Sander JW. Epilepsy in adults. Lancet. 2019;393(10172):689-701. doi:10.1016/S0140-6736(18) 32596-0

107. Gavvala J, Zafar M, Sinha SR, Kalamangalam G, Schuele S. Stereotactic EEG practices: a survey of United States tertiary referral epilepsy centers. J Clin Neurophysiol. 2020. doi:10.1097/WNP.0000000000000794

108. Ryvlin P, Rheims S. Predicting epilepsy surgery outcome. Curr Opin Neurol. 2016;29(2):182-188. doi:10.1097/ WCO.0000000000000306

109. Jain P, Smith ML, Speechley K, et al. Seizure freedom improves health-related quality of life after epilepsy surgery in children. Dev Med Child Neurol. 2020;62(5):600-608. doi:10.1111/ dmcn. 14390

110. Ramos-Perdigués S, Baillés E, Mané A, et al. A prospective study contrasting the psychiatric outcome in drug-resistant epilepsy between patients who underwent surgery and a control group. Epilepsia. 2016;57(10):1680-1690. doi:10.1111/epi.13497

111. Morrison-Levy N, Go C, Ochi A, et al. Children with autism spectrum disorders and drug-resistant epilepsy can benefit from epilepsy surgery. Epilepsy Behavior. 2018;85:200-204. doi:10.1016/j.yebeh.2018.06.023

112. Lang JD, Grell L, Hagge M, et al. Long-term outcome after epilepsy surgery in older adults. Seizure. 2018;57:56-62. doi:10.1016/j.seizure.2018.02.012

113. Lazow SP, Thadani VM, Gilbert KL, et al. Outcome of frontal lobe epilepsy surgery: outcome of Frontal Lobe Epilepsy Surgery. Epilepsia. 2012;53(10):1746-1755. doi:10.1111/j.15281167.2012.03582.x

114. Chassoux F, Landré E, Mellerio C, et al. Type II focal cortical dysplasia: electroclinical phenotype and surgical outcome related to imaging: phenotype and Imaging in TTFCD. Epilepsia. 2012;53(2):349-358. doi:10.1111/j.1528 1167.2011.03363.x

115. Kang JY, Sperling MR. Magnetic resonance imaging-guided laser interstitial thermal therapy for treatment of drug-resistant epilepsy. Neurotherapeutics. 2017;14(1):176-181. doi:10.1007/ s13311-016-0498-3

116. Shimamoto S, Wu C, Sperling MR. Laser interstitial thermal therapy in drug-resistant epilepsy. Curr Opin Neurol. 2019;32 (2):237-245. doi:10.1097/WCO.0000000000000662
117. Bourdillon $\mathrm{P}$, Cucherat $\mathrm{M}$, Isnard $\mathrm{J}$, et al. Stereoelectroencephalography-guided radiofrequency thermocoagulation in patients with focal epilepsy: a systematic review and meta-analysis. Epilepsia. 2018;59(12):2296-2304. doi:10.1111/epi.14584

118. Benbadis SR, Geller E, Ryvlin P, et al. Putting it all together: options for intractable epilepsy. Epilepsy Behavior. 2018;88:33-38. doi:10.1016/j.yebeh.2018.05.030

119. Orosz I, McCormick D, Zamponi N, et al. Vagus nerve stimulation for drug-resistant epilepsy: a European long-term study up to 24 months in 347 children. Epilepsia. 2014;55(10):1576-1584. doi:10.1111/epi.12762

120. Ryvlin P, Gilliam FG, Nguyen DK, et al. The long-term effect of vagus nerve stimulation on quality of life in patients with pharmacoresistant focal epilepsy: the PuLsE (Open Prospective Randomized Long-term Effectiveness) trial. Epilepsia. 2014;55 (6):893-900. doi:10.1111/epi.12611

121. Bouwens van der Vlis TAM, Schijns OEMG, Schaper FLWVJ, et al. Deep brain stimulation of the anterior nucleus of the thalamus for drug-resistant epilepsy. Neurosurg Rev. 2019;42 (2):287-296. doi:10.1007/s10143-017-0941-x

122. Salanova V, Witt $T$, Worth $R$, et al. Long-term efficacy and safety of thalamic stimulation for drug-resistant partial epilepsy. Neurology. 2015;84(10):1017-1025. doi:10.1212/ WNL.0000000000001334

123. Velasco AL, Velasco F, Jimenez F, et al. Neuromodulation of the centromedian thalamic nuclei in the treatment of generalized seizures and the improvement of the quality of life in patients with lennox-gastaut syndrome. Epilepsia. 2006;47(7):1203-1212. doi:10.1111/j.1528-1167.2006.00593.x

124. Skarpaas TL, Jarosiewicz B, Morrell MJ. Brain-responsive neurostimulation for epilepsy $\left(\mathrm{RNS}^{\circledR}\right.$ System). Epilepsy Res. 2019;153:68-70. doi:10.1016/j.eplepsyres.2019.02.003

125. Nune G, Arcot Desai S, Razavi B, et al. Treatment of drugresistant epilepsy in patients with periventricular nodular heterotopia using RNS $^{\circledR}$ System: efficacy and description of chronic electrophysiological recordings. Clin Neurophysiol. 2019;130 (8):1196-1207. doi:10.1016/j.clinph.2019.04.706

126. Lambrechts DAJE, de Kinderen RJA, Vles JSH, de Louw AJA, Aldenkamp AP, Majoie HJM. A randomized controlled trial of the ketogenic diet in refractory childhood epilepsy. Acta Neurol Scand. 2017;135(2):231-239. doi:10.1111/ane.12592

127. Martin-McGill KJ, Bresnahan R, Levy RG, Cooper PN. Ketogenic diets for drug-resistant epilepsy. Cochrane Database Systematic Rev. 2020. doi:10.1002/14651858.CD001903.pub5

128. Kverneland M, Taubøll E, Molteberg E, et al. Pharmacokinetic interaction between modified Atkins diet and antiepileptic drugs in adults with drug-resistant epilepsy. Epilepsia. 2019;60 (11):2235-2244. doi:10.1111/epi.16364

129. Gómez-Eguílaz M, Ramón-Trapero JL, Pérez-Martínez L, Blanco JR. The beneficial effect of probiotics as a supplementary treatment in drug-resistant epilepsy: a pilot study. Benef Microbes. 2018;9 (6):875-881. doi:10.3920/BM2018.0018

130. Leidy NK, Elixhauser A, Vickrey B, Means E, Willian MK. Seizure frequency and the health-related quality of life of adults with epilepsy. Neurology. 1999;53(1):162. doi:10.1212/WNL.53.1.162

131. Jacoby A, Baker GA, Steen N, Potts P, Chadwick DW. The clinical course of epilepsy and its psychosocial correlates: findings from a U.K. Community Study. Epilepsia. 1996;37 (2):148-161. doi:10.1111/j.1528-1157.1996.tb00006.x

132. Guekht AB, Mitrokhina TV, Lebedeva AV, et al. Factors influencing on quality of life in people with epilepsy. Seizure. 2007;16 (2):128-133. doi:10.1016/j.seizure.2006.10.011

133. Johnson EK, Jones JE, Seidenberg M, Hermann BP. The relative impact of anxiety, depression, and clinical seizure features on health-related quality of life in epilepsy. Epilepsia. 2004;45 (5):544-550. doi:10.1111/j.0013-9580.2004.47003.x 
134. Cramer J. A systematic review of the behavioral effects of levetiracetam in adults with epilepsy, cognitive disorders, or an anxiety disorder during clinical trials. Epilepsy Behavior. 2003;4 (2):124-132. doi:10.1016/S1525-5050(03)00005-2

135. Sheikh SR, Thompson N, Frech F, Malhotra M, Jehi L. Quantifying the burden of generalized tonic-clonic seizures in patients with drug-resistant epilepsy. Epilepsia. 2020;61 (8):1627-1637. doi:10.1111/epi.16603

136. Sherman EMS, Slick DJ, Eyrl KL. Executive dysfunction is a significant predictor of poor quality of life in children with epilepsy. Epilepsia. 2006;47(11):1936-1942. doi:10.1111/j.15281167.2006.00816.x

137. Giovagnoli AR, Parente A, Tarallo A, Casazza M, Franceschetti S, Avanzini G. Self-rated and assessed cognitive functions in epilepsy: impact on quality of life. Epilepsy Res. 2014;108(8):1461-1468. doi:10.1016/j.eplepsyres.2014.06.002

138. Sarkis RA, McGinnis S, Rushia SN, Park S, Ansari EE, Willment KC. Growing older with drug-resistant epilepsy: cognitive and psychosocial outcomes. $J$ Neurol. 2018;265 (5):1059-1064. doi:10.1007/s00415-018-8805-z

139. Camfield PR, Andrade D, Camfield CS, et al. How can transition to adult care be best orchestrated for adolescents with epilepsy? Epilepsy Behavior. 2019;93:138-147. doi:10.1016/j. yebeh.2018.12.015

140. Tellez-Zenteno JF, Patten SB, Jetté N, Williams J, Wiebe S. Psychiatric comorbidity in epilepsy: a population-based analysis. Epilepsia. 2007. doi:10.1111/j.1528-1167.2007.01222.x

141. Christensen J, Vestergaard M, Mortensen PB, Sidenius P, Agerbo E. Epilepsy and risk of suicide: a population-based case-control study. Lancet Neurol. 2007;6(8):693-698. doi:10.1016/S1474-4422(07)70175-8
142. Beyenburg S, Mitchell AJ, Schmidt D, Elger CE, Reuber M. Anxiety in patients with epilepsy: systematic review and suggestions for clinical management. Epilepsy Behavior. 2005;7 (2):161-171. doi:10.1016/j.yebeh.2005.05.014

143. Almanza-Sepulveda ML, Smith ML, Widjaja E. Influence of patient, caregiver, and family factors on unmet healthcare needs in children with drug-resistant epilepsy undergoing epilepsy surgery evaluation. Epilepsy Behavior. 2019;99:106502. doi:10.1016/j.yebeh.2019.106502

144. Gilliam FG, Barry JJ, Hermann BP, Meador KJ, Vahle V, Kanner AM. Rapid detection of major depression in epilepsy: a multicentre study. Lancet Neurol. 2006;5(5):399-405. doi:10.1016/S1474-4422(06)70415-X

145. Kanner AM. Most antidepressant drugs are safe for patients with epilepsy at therapeutic doses: a review of the evidence. Epilepsy Behavior. 2016;61:282-286. doi:10.1016/j.yebeh.2016.03.022

146. Conway CR, Udaiyar A, Schachter SC. Neurostimulation for depression in epilepsy. Epilepsy Behavior. 2018;88:25-32. doi:10.1016/j.yebeh.2018.06.007

147. Tang V, Poon WS, Kwan P. Mindfulness-based therapy for drug-resistant epilepsy: an assessor-blinded randomized trial. Neurology. 2015;85(13):1100-1107. doi:10.1212/ WNL.0000000000001967

148. Wilson S. the Task Force members. In response: indications and expectations for neuropsychological assessment in routine epilepsy care: report of the ILAE Neuropsychology Task Force, Diagnostic Methods Commission, 2013-2017. Epilepsia. 2015;56(8):1316-1317. doi:10.1111/epi.13055

\section{Publish your work in this journal}

Neuropsychiatric Disease and Treatment is an international, peerreviewed journal of clinical therapeutics and pharmacology focusing on concise rapid reporting of clinical or pre-clinical studies on a range of neuropsychiatric and neurological disorders. This journal is indexed on PubMed Central, the 'PsycINFO' database and CAS, and is the official journal of The International Neuropsychiatric Association (INA). The manuscript management system is completely online and includes a very quick and fair peer-review system, which is all easy to use. Visit http://www.dovepress.com/testimonials.php to read real quotes from published authors. 